\title{
Solvability for a class of evolution equations of fractional order with nonlocal conditions on the half-line
}

\section{Zhanmei Lv ${ }^{1}$, Yanping Gong ${ }^{1 *}$ and Yi Chen ${ }^{2}$}

"Correspondence:
gongyp2012@sina.com
${ }^{1}$ School of Business, Central South
University, Changsha, 410083, China
Full list of author information is
available at the end of the article

available at the end of the article

\begin{abstract}
In this paper, we get a new form equivalent integral equation for a class of evolution equations of fractional order with nonlocal conditions on the half-line. With the aid of it, the uniqueness of the mild solution is obtained by the Banach contraction theorem. Also, we present the existence and uniqueness theorem of positive mild solutions by the monotone iterative method without assumption of lower and upper solutions.

Keywords: fractional order; abstract evolution equations; mild solutions; monotone iterative method
\end{abstract}

\section{Introduction}

In this paper, we deal with the following functional differential abstract equation of fractional order with nonlocal conditions in the Banach space $E$ :

$$
\left\{\begin{array}{l}
{ }^{C} D_{0+}^{q} u(t)=A u(t)+f(t, u(r(t))), \quad t \in(0,+\infty), \\
u(0)=\sum_{i=1}^{\infty} \sigma_{i} u\left(\tau_{i}\right),
\end{array}\right.
$$

where ${ }^{C} D_{0+}^{q}$ is the Caputo fractional derivative, $0<q<1, \sigma_{i}>0, \tau_{i}>0(i=1,2, \ldots), A$ is the infinitesimal generator of a $C_{0}$ semigroup $\{T(t)\}_{t \geq 0}$ of operators on Banach $E, r \in C[0, \infty)$, $r(t) \geq 0$, and $f:[0,+\infty) \times E \rightarrow E$ satisfies certain conditions.

If $r(t)=t$, then problem (1) can be written as

$$
\left\{\begin{array}{l}
{ }^{C} D_{0+}^{q} u(t)=A u(t)+f(t, u(t)), \quad t \in(0,+\infty), \\
u(0)=\sum_{i=1}^{\infty} \sigma_{i} u\left(\tau_{i}\right)
\end{array}\right.
$$

Fractional calculus, a generalization of the ordinary differentiation and integration, has played an important role in science, biology, physics, economy, engineering, and other fields (see [1-3]). A large number of phenomena and processes in the real world are described by differential equation of fractional order, due to the fact of its various applications in many areas. Today, there are a lot of researchers committed to investigating the

(c) The Author(s) 2017. This article is distributed under the terms of the Creative Commons Attribution 4.0 International License (http://creativecommons.org/licenses/by/4.0/), which permits unrestricted use, distribution, and reproduction in any medium, provided you give appropriate credit to the original author(s) and the source, provide a link to the Creative Commons license, and indicate if changes were made. 
fractional differential equations. For more general theory of fractional differential equations, we refer readers to the papers [4-23] and the references given therein.

In [14], EI-Sayed obtained the existence and uniqueness results based on an equivalent integral equation for a class of fractional evolution equations, which was described in terms of some probability densities by the method of Laplace transform for the first time. Since then, according to EI-Sayed's results, most papers have been working on the evolution equations of fractional order, see [15-23].

Now, one branch of the studies on fractional differential equations is devoted to investigating the fractional evolution equation with nonlocal conditions, which is a valuable tool to describe the physics phenomena. Nonlocal conditions were initiated by Byszewski [24] when he gave the existence and uniqueness results of the mild solutions for the nonlocal Cauchy problems. In [25], Byszewski and Lakshmikantham indicated that the nonlocal condition can be more useful than the standard initial condition to model some physical phenomena.

In [26], Byszewski dealt with the following functional-differential abstract nonlocal Cauchy problem of integer order in a general Banach space:

$$
\left\{\begin{array}{l}
u^{\prime}(t)=f(t, u(t), u(a(t))), \quad t \in\left[t_{0}, t_{0}+T\right], \\
u\left(t_{0}\right)+\sum_{k=1}^{p} c_{k} u\left(t_{k}\right)=x_{0} .
\end{array}\right.
$$

Applying the Banach contraction theorem and a modified Picard method, the existence and uniqueness of a classical solution is given.

In [27], Chen studied a class of nonlocal evolution equations

$$
\left\{\begin{array}{l}
u^{\prime}(t)+A u(t)=f(t, u(t)), \quad t \geq 0, \\
u(0)=\sum_{k=1}^{\infty} c_{k} u\left(t_{k}\right) .
\end{array}\right.
$$

With the help of the monotone iterative method, the existence and uniqueness of the mild positive solutions were obtained in the paper.

As far as we know, recently, evolution equations of fractional order have attracted increasing attention, and we refer to the papers [13-23] and the references therein. However, many of those papers on the existence of solutions of fractional evolution equations are on the finite interval, and the existence results on the half-line are still few.

Motivated by the papers $[14,15,26,27]$, in this paper, we study the fractional differential equations (1) and (2) with nonlocal conditions on the unbounded domains. Here, we give a corrected form of the equivalent integral equation of (1) by similar methods as the ones used in $[14,15]$, which is different from those obtained in the existing literature. With the aid of the Banach contraction theorem, the uniqueness of the mild solution of problem (1) is given. Besides, employing the monotone iterative method, without the assumption of lower and upper solutions, we present some new results on the existence of positive mild solutions for the abstract fractional differential equations (2).

For the sake of convenience, we denote

$$
\chi:=\frac{1}{\Gamma(q+1)} \frac{1}{1-\sum_{i=1}^{\infty} \sigma_{i}} .
$$

Our main results are as follows. 
Theorem 1.1 Let $E$ be a Banach space and $\{T(t)\}_{t \geq 0}$ be a uniformly exponentially stable $C_{0}$-semigroup with the growth bound $\omega_{0}\left(\omega_{0}<0\right)$, and $A$ is the infinitesimal generator of $\{T(t)\}_{t \geq 0}$. Let $0<\sum_{i=1}^{\infty} \sigma_{i}<1$. Let $r \in C[0, \infty)$ and $r(t) \geq 0$. If $f(t, u)$ satisfies the following condition:

(F) There exist two constants $\omega^{*} \in\left(0,\left|\omega_{0}\right|\right]$ and $\mathcal{M}$ satisfying $\mathcal{M}<\omega^{*} / \chi$ such that

$$
\|f(t, x)-f(t, y)\|_{\omega^{*}} \leq \mathcal{M}\|x-y\|_{\omega^{*}}, \quad \forall x, y \in E,
$$

then problem (1) has a unique mild solution in $B C(J, E)$. Besides, iff $(t, \theta) \neq \theta$, the unique mild solution in $B(J, E)$ is nontrivial.

Theorem 1.2 Let $E$ be a Banach space, and $P$ is its positive normal with $N$ as the normal constant. Let $\{T(t)\}_{t \geq 0}$ be a uniformly exponentially stable $C_{0}$-semigroup with the growth bound $\omega_{0}\left(\omega_{0}<0\right)$, and $A$ is the infinitesimal generator of $\{T(t)\}_{t \geq 0}$. Let $0<\sum_{i=1}^{\infty} \sigma_{i}<1$. Provided that $f(t, u): J \times E \longrightarrow E$ is continuous and $f_{0}(t):=f(t, \theta) \geq \theta$ is bounded on $J$, if $f(t, u)$ satisfies the following conditions:

(H1) There exist two constants $d_{1}<-\omega_{0}$ and $d_{2}>\max \left\{-d_{1}, \omega_{0}\right\}$ such that for

$$
-d_{2}(y-x) \leq f(t, y)-f(t, x) \leq d_{1}(y-x), \quad \theta \leq x \leq y
$$

$$
0<\frac{d_{1}+d_{2}}{d_{2}-\omega_{0}}<\frac{1}{\chi}
$$

then problem (2) has a unique positive mild solution in $B C(J, E)$.

Remark 1.1 We give a comparison between the two methods. First, the existence of positive mild solution is given in Theorem 1.1. However, Theorem 1.2 only shows the existence of mild solution, and it cannot tell if it is positive. Second, the function $f(t, x)$ should be a monotone one in Theorem 1.2, but this condition is not necessary in Theorem 1.1. Given the above, these two theorems are complementary.

The rest of the paper is organized as follows. In Section 2, we introduce the definitions of the fractional integral and fractional derivative, some results about fractional differential equations and some useful preliminaries. In Section 3, we present the proofs of our main results by the Banach contraction theorem and the monotone iterative method, respectively. Then an example is given in Section 4 to demonstrate the application of our results.

\section{Preliminaries}

First of all, we present some fundamental facts on the fractional calculus theory which we will use in the next section. 
Definition 2.1 ([1-3]) The Riemann-Liouville fractional integral of order $v>0$ of a function $h:(0, \infty) \rightarrow \mathbb{R}$ is given by

$$
I_{0+}^{v} h(t)=D_{0+}^{-v} h(t)=\frac{1}{\Gamma(v)} \int_{0}^{t}(t-s)^{\nu-1} h(s) d s,
$$

provided that the right-hand side is pointwise defined on $(0, \infty)$.

Definition $2.2([1-3])$ The Caputo fractional derivative of order $v>0$ of a continuous function $h:(0, \infty) \rightarrow \mathbb{R}$ is given by

$$
{ }^{C} D_{0+}^{v} h(t)=\frac{1}{\Gamma(n-v)} \int_{0}^{t}(t-s)^{n-v-1} h^{n}(s) d s
$$

where $n=[v]+1$, provided that the right-hand side is pointwise defined on $(0, \infty)$.

Lemma 2.1 ([1, 3]) Assume that ${ }^{C} D_{0+}^{v} h(t) \in L^{1}(0,+\infty), v>0$. Then we have

$$
I_{0+}^{v}{ }^{C} D_{0+}^{v} h(t)=h(t)+C_{1}+C_{2} t+\cdots+C_{N} t^{N-1}, \quad t>0,
$$

for some $C_{i} \in \mathbb{R}, i=1,2, \ldots, N$,where $N$ is the smallest integer greater than or equal to $v$.

If $h$ is an abstract function with values in the Banach space $E$, then the integrals appearing in Definition 2.1, Definition 2.2 and Lemma 2.1 are taken in Bochner's sense. And a measurable function $h$ is Bochner integrable if the norm of $h$ is Lebesgue integrable.

Now let us recall some definitions and standard facts about the cone.

Let $P$ be a cone in the ordered Banach space $E$, which defines a partial order on $E$ by $x \leq y$ if and only if $y-x \in P$. $P$ is normal if there exists a positive constant $N$ such that $\theta \leq x \leq y$ implies $\|x\| \leq N\|y\|$, where $\theta$ is the zero element of the Banach space $E$. The infimum of all $N$ with the property above is called the normal constant of $P$. For more details on the cone $P$, we refer the readers to $[28,29]$.

Throughout the paper, we set $E$ be an ordered Banach space with the norm $\|\cdot\|$ and the partial order ' $\leq$. Let $P=\{x \in E \mid x \geq \theta\}$ be a positive cone, which is normal with normal constant $N$. Let $J=[0,+\infty)$. Set

$$
B C(J, E)=\{u(t) \mid u(t) \text { is continuous and bounded on } J\} .
$$

Obviously, $B C(J, E)$ is a Banach space with the norm $\|u\|_{b}=\sup _{t \in J}\|u(t)\|$. Let

$$
P_{B}=\{u \in B C(J, E) \mid u(t) \geq \theta, t \in J\} .
$$

It is easy to see that $P_{B}$ is also normal with the same normal constant $N$ of the cone $P$. Besides, $B C(J, E)$ is also an ordered Banach space with the partial order ' $\leq$ ' induced by the positive cone $P_{B}$ (without confusion, we denote by ' $\leq$ ' the partial order on both $E$ and $B C(J, E))$.

We denote by $[v, w]$ the order interval $\left\{u \in P_{B} \mid v \leq u \leq w, v, w \in B C(J, E)\right\}$ on $B C(J, E)$, and use $[v(t), w(t)]$ to denote the order interval $\{z \in E \mid v(t) \leq z \leq w(t)\}$ on $E$ for $t \in J$. 
Next, we give some facts about the semigroups of linear operators. These results can be found in [30, 31].

For a strongly continuous semigroup (i.e., $C_{0}$-semigroup) $\{T(t)\}_{t \geq 0}$, the infinitesimal generator of $\{T(t)\}_{t \geq 0}$ is defined by

$$
A x=\lim _{t \rightarrow 0^{+}} \frac{T(t) x-x}{t}, \quad x \in E .
$$

We denote by $D(A)$ the domain of $A$, that is,

$$
D(A)=\left\{x \in E \mid \lim _{t \rightarrow 0^{+}} \frac{T(t) x-x}{t} \text { exists }\right\} .
$$

Lemma $2.2([30,31])$ Let $\{T(t)\}_{t \geq 0}$ be a $C_{0}$-semigroup, then there exist constants $C \geq 1$ and $\omega \in \mathbb{R}$ such that $\|T(t)\| \leq C e^{\omega t}, t \geq 0$.

Lemma $2.3([30,31])$ A linear operator $A$ is the infinitesimal generator of a $C_{0}$-semigroup $\{T(t)\}_{t \geq 0}$ if and only if

(i) $A$ is closed and $\overline{D(A)}=E$.

(ii) The resolvent set $\rho(A)$ of $A$ contains $\mathbb{R}^{+}$and, for every $\lambda>0$, we have

$$
\|R(\lambda, A)\| \leq \frac{1}{\lambda}
$$

where

$$
R(\lambda, A):=(\lambda I-A)^{-1}=\int_{0}^{+\infty} e^{-\lambda t} T(t) x d t, \quad x \in E .
$$

Definition 2.3 ([30, 31]) A $C_{0}$-semigroup $\{T(t)\}_{t \geq 0}$ is said to be uniformly exponentially stable if $\omega_{0}<0$, where $\omega_{0}$ is the growth bound of $\{T(t)\}_{t \geq 0}$, which is defined by

$$
\omega_{0}=\inf \left\{\omega \in \mathbb{R} \mid \exists C \geq 1 \text { such that }\|T(t)\| \leq C e^{\omega t}, t \geq 0\right\} .
$$

Definition 2.4 ([27]) A $C_{0}$-semigroup $\{T(t)\}_{t \geq 0}$ is said to be positive on $E$, if order inequality $T(t) x \geq \theta, x \in E$ and $t \geq 0$.

According to Lemma 2.2 and Definition 2.3, if $\{T(t)\}_{t \geq 0}$ is a uniformly exponentially stable $C_{0}$-semigroup with the growth bound $\omega_{0}$, then for any $\omega \in\left(0,\left|\omega_{0}\right|\right]$, there exists a constant $C \geq 1$ such that $\|T(t)\| \leq C e^{\omega t}, t \geq 0$. Now, we define a norm in $E$ by

$$
\|x\|_{\omega}=\sup _{t \geq 0}\left\|e^{\omega t} T(t) x\right\|
$$

Evidently, we have $\|x\| \leq\|x\|_{\omega} \leq C\|x\|$, that is to say, the norm $\|\cdot\|_{\omega}$ and $\|\cdot\|$ are equivalent. We denote by $\|T(t)\|_{\omega}$ the norm of $T(t)$ induced by the norm $\|\cdot\|_{\omega}$. Then we obtain

$$
\|T(t)\|_{\omega} \leq e^{-\omega t}, \quad t \geq 0
$$


Also, we can define the equivalent norm on $B C(J, E)$ by

$$
\|u\|_{b \omega}=\sup _{t \in J}\|u(t)\|_{\omega}, \quad u \in B C(J, E) .
$$

Obviously, if $u(t) \equiv u_{0}, t \in J, u_{0} \in E$, then we have

$$
\|u\|_{b \omega}=\left\|u_{0}\right\|_{b \omega}=\|u\|_{\omega}
$$

Consider the one-sided stable probability density $[15,16,32]$

$$
\psi_{q}(\theta)=\frac{1}{\pi} \sum_{n=1}^{\infty} \theta^{-q n-1} \frac{\Gamma(n q+1)}{n !} \sin (n \pi q), \quad \theta \in(0, \infty)
$$

where $0<q<1$. By Remark 2.8 in [16], for $0 \leq \gamma \leq 1$, one has

$$
\int_{0}^{\infty} \theta^{-q \gamma} \psi_{q}(\theta) d \theta=\frac{\Gamma(1+\gamma)}{\Gamma(1+q \gamma)}
$$

From $[15,16,32]$, the Laplace transform of the one-sided stable probability density $\psi_{q}(\theta)$ is given by

$$
\mathcal{L}\left[\psi_{q}(\theta)\right]=\int_{0}^{\infty} e^{-\lambda \theta} \psi_{q}(\theta) d \theta=e^{-\lambda^{q}}, \quad 0<q<1 .
$$

In the following, we assume that $\{T(t)\}_{t \geq 0}$ is a uniformly exponentially stable $C_{0^{-}}$ semigroup with the growth bound $\omega_{0}$, and $\omega \in\left(0,\left|\omega_{0}\right|\right)$.

\section{Lemma 2.4 Define an operator}

$$
\begin{aligned}
& (\Upsilon h)(t):=\int_{0}^{1} \int_{0}^{\infty} \frac{q}{\Gamma(1-q)} \frac{\psi_{q}(\theta)}{\theta^{q}} \tau^{-q}(1-\tau)^{q-1} T\left(\frac{t^{q}(1-\tau)^{q}}{\theta^{q}}\right) h(s) d \theta d \tau, \\
& h \in B C(J, E) .
\end{aligned}
$$

Then $\Upsilon: B C(J, E) \longrightarrow B C(J, E)$ and

$$
\|\Upsilon h\|_{b \omega} \leq\|h\|_{b \omega} .
$$

In particular, if $h(t) \equiv x, t \in J, x \in E$, then

$$
\|\Upsilon x\|_{b \omega} \leq\|x\|_{\omega}
$$

Proof Since

$$
\begin{aligned}
& \|(\Upsilon h)(t)\|_{\omega} \\
& \quad \leq \int_{0}^{1} \int_{0}^{\infty} \frac{q}{\Gamma(1-q)} \tau^{-q}(1-\tau)^{q-1} \frac{\psi_{q}(\theta)}{\theta^{q}}\left\|T\left(\frac{t^{q}(1-\tau)^{q}}{\theta^{q}}\right) h(s)\right\|_{\omega} d \theta d \tau
\end{aligned}
$$




$$
\begin{aligned}
& \leq \int_{0}^{1} \int_{0}^{\infty} \frac{q}{\Gamma(1-q)} \tau^{-q}(1-\tau)^{q-1} \frac{\psi_{q}(\theta)}{\theta^{q}}\left\|T\left(\frac{t^{q}(1-\tau)^{q}}{\theta^{q}}\right)\right\|_{\omega}\|h(s)\|_{\omega} d \theta d \tau \\
& \leq \int_{0}^{1} \int_{0}^{\infty} \frac{q}{\Gamma(1-q)} \tau^{-q}(1-\tau)^{q-1} \frac{\psi_{q}(\theta)}{\theta^{q}} e^{-\omega\left(\frac{t^{q(1-\tau)^{q}}}{\theta^{q}}\right)}\|h\|_{b \omega} d \theta d \tau \\
& \leq \frac{q}{\Gamma(1-q)}\|h\|_{b \omega} \int_{0}^{1} \tau^{-q}(1-\tau)^{q-1}\left(\int_{0}^{\infty} \frac{\psi_{q}(\theta)}{\theta^{q}} d \theta\right) d \tau \\
& \leq\|h\|_{b \omega},
\end{aligned}
$$

then the proof is finished.

Lemma 2.5 Define a linear operator $\mathcal{R}: B C(J, E) \longrightarrow B C(J, E)$ as

$$
\begin{aligned}
\mathcal{R} h & :=\sum_{i=1}^{\infty} \sigma_{i}\left[(\Upsilon h)\left(\tau_{i}\right)\right] \\
& =\sum_{i=1}^{\infty} \sigma_{i} \int_{0}^{1} \int_{0}^{\infty} \frac{q}{\Gamma(1-q)} \frac{\psi_{q}(\theta)}{\theta^{q}} \tau^{-q}(1-\tau)^{q-1} T\left(\tau_{i}^{q} \frac{(1-\tau)^{q}}{\theta^{q}}\right) x d \theta d \tau
\end{aligned}
$$

Then $\mathcal{R}$ is bounded and $\|\mathcal{R}\|_{\omega} \leq \sum_{i=1}^{\infty} \sigma_{i}$. Besides, if $0<\sum_{i=1}^{\infty} \sigma_{i}<1$, then $(I-\mathcal{R})^{-1}$ is a linear bounded operator and

$$
\left\|(I-\mathcal{R})^{-1}\right\|_{\omega} \leq \frac{1}{1-\sum_{i=1}^{\infty} \sigma_{i}} .
$$

Proof In view of Lemma 2.4, one can get

$$
\begin{aligned}
\|\mathcal{R} h\|_{\omega} & =\left\|\sum_{i=1}^{\infty} \sigma_{i}\left[(\Upsilon h)\left(\tau_{i}\right)\right]\right\|_{\omega} \leq \sum_{i=1}^{\infty} \sigma_{i}\left\|\left[(\Upsilon h)\left(\tau_{i}\right)\right]\right\|_{\omega} \leq \sum_{i=1}^{\infty} \sigma_{i}\|\Upsilon h\|_{b \omega} \\
& \leq\left(\sum_{i=1}^{\infty} \sigma_{i}\right)\|h\|_{\omega} .
\end{aligned}
$$

Hence, $\mathcal{R}$ is bounded and $\|\mathcal{R}\|_{\omega} \leq \sum_{i=1}^{\infty} \sigma_{i}$.

Lemma 2.6 Set

$$
(\mathcal{W h})(t)=\int_{0}^{t} \int_{0}^{\infty} q \frac{\psi_{q}(\theta)}{\theta^{q}}(t-s)^{q-1} T\left(\frac{(t-s)^{q}}{\theta^{q}}\right) h(s) d \theta d s, \quad h \in B C(J, E) .
$$

Then $\mathcal{W}: B C(J, E) \longrightarrow B C(J, E)$ and

$$
\|(\mathcal{W} h)(t)\|_{\omega} \leq \frac{1}{\omega} \frac{1}{\Gamma(q+1)}\|h\|_{b \omega} ; \quad\|(\mathcal{W} h)\|_{b \omega} \leq \frac{1}{\omega} \frac{1}{\Gamma(q+1)}\|h\|_{b \omega} .
$$

Proof Since

$$
\begin{aligned}
(\mathcal{W h})(t) & =\int_{0}^{t} \int_{0}^{\infty} q \frac{\psi_{q}(\theta)}{\theta^{q}}(t-s)^{q-1} T\left(\frac{(t-s)^{q}}{\theta^{q}}\right) h(s) d \theta d s \\
& =\int_{0}^{1} \int_{0}^{\infty} q \frac{\psi_{q}(\theta)}{\theta^{q}} t^{q}(1-\tau)^{q-1} T\left(\frac{t^{q}(1-\tau)^{q}}{\theta^{q}}\right) h(t \tau) d \theta d \tau
\end{aligned}
$$


then

$$
\begin{aligned}
& \|(\mathcal{W} h)(t)\|_{\omega} \\
& \leq \int_{0}^{1} \int_{0}^{\infty} q \frac{\psi_{q}(\theta)}{\theta^{q}} t^{q}(1-\tau)^{q-1}\left\|T\left(\frac{t^{q}(1-\tau)^{q}}{\theta^{q}}\right)\right\|_{\omega}\|h(t \tau)\|_{\omega} d \theta d \tau \\
& \leq \int_{0}^{1} \int_{0}^{\infty} q \frac{\psi_{q}(\theta)}{\theta^{q}} t^{q}(1-\tau)^{q-1} e^{-\omega \frac{t^{q(1-\tau)}}{\theta^{q}}}\|h(t \tau)\|_{\omega} d \theta d \tau \\
& \leq \frac{1}{\omega}\|h\|_{b \omega} \int_{0}^{\infty}\left[\left(\int_{0}^{1} e^{\left.-\omega \frac{t^{q}(1-\tau)^{q}}{\theta^{q}}\right)} d\left(-\omega \frac{t^{q}(1-\tau)^{q}}{\theta^{q}}\right)\right) \frac{\psi_{q}(\theta)}{\theta^{q}}\right] d \theta \\
& =\frac{1}{\omega}\|h\|_{b \omega} \int_{0}^{\infty}\left(1-e^{-\omega \frac{t^{q}}{\theta^{q}}}\right) \frac{\psi_{q}(\theta)}{\theta^{q}} d \theta \\
& \leq \frac{1}{\omega} \frac{1}{\Gamma(q+1)}\|h\|_{b \omega} .
\end{aligned}
$$

Therefore,

$$
\|(\mathcal{W} h)\|_{b \omega} \leq \frac{1}{\omega} \frac{1}{\Gamma(q+1)}\|h\|_{b \omega} .
$$

Lemma 2.7 Let $h \in B C(J, E)$ and $u_{0} \in D(A)$. Assume that $0<\sum_{i=1}^{\infty} \sigma_{i}<1$. Then the linear fractional evolution equation

$$
\left\{\begin{array}{l}
{ }^{C} D_{0+}^{q} u(t)=A u(t)+h(t), \quad t \in(0,+\infty), \\
u(0)=u_{0}
\end{array}\right.
$$

has a unique solution $u \in B C(J, E)$ of the following form:

$$
\begin{aligned}
u(t)= & \left(\Upsilon u_{0}\right)(t)+(\mathcal{W h})(t) \\
= & \int_{0}^{1} \int_{0}^{\infty} \frac{q}{\Gamma(1-q)} \frac{\psi_{q}(\theta)}{\theta^{q}} \tau^{-q}(1-\tau)^{q-1} T\left(\frac{t^{q}(1-\tau)^{q}}{\theta^{q}}\right) u_{0} d \theta d \tau \\
& +\int_{0}^{t} \int_{0}^{\infty} q \frac{\psi_{q}(\theta)}{\theta^{q}}(t-s)^{q-1} T\left(\frac{(t-s)^{q}}{\theta^{q}}\right) h(s) d \theta d s .
\end{aligned}
$$

Proof In view of Definitions 2.1, 2.2 and Lemma 2.1, equation (10) can be rewritten by the equivalent integral equation as follows:

$$
u(t)=u_{0}+\frac{1}{\Gamma(q)} \int_{0}^{t}(t-s)^{q-1}[A u(s)+h(s)] d s .
$$

Denote by $U(\lambda)$ and $H(\lambda)$ the Laplace transform of $u(t)$ and $h(t)$, respectively, using a similar method as that in $[13,14]$, then with the Laplace transform, we can rewrite the above equation as

$$
U(\lambda)=\frac{1}{\lambda} u_{0}+\frac{1}{\lambda^{q}} A U(\lambda)+\frac{1}{\lambda^{q}} H(\lambda), \quad \lambda>0 .
$$

Then one has

$$
\left(\lambda^{q} I-A\right) U(\lambda)=\lambda^{q-1} u_{0}+H(\lambda) .
$$


By virtue of (8) and Lemma 2.3, we obtain

$$
\begin{aligned}
U(\lambda)= & \left(\lambda^{q} I-A\right)^{-1} \lambda^{q-1} u_{0}+\left(\lambda^{q} I-A\right)^{-1} H(\lambda) \\
= & \lambda^{q-1} \int_{0}^{\infty} e^{-\lambda^{q} s} T(s) u_{0} d s+\int_{0}^{\infty} e^{-\lambda^{q} s} T(s) H(\lambda) d s \\
= & \lambda^{q-1} \int_{0}^{\infty} \int_{0}^{\infty} e^{-\lambda s^{1 / q} \theta} \psi_{q}(\theta) T(s) u_{0} d \theta d s \\
& +\int_{0}^{\infty} \int_{0}^{\infty} e^{-\lambda s^{1 / q} \theta} \psi_{q}(\theta) T(s) H(\lambda) d \theta d s \\
= & \lambda^{q-1} \int_{0}^{\infty} e^{-\lambda t}\left[\int_{0}^{\infty} q \frac{t^{q-1}}{\theta^{q}} \psi_{q}(\theta) T\left(\frac{t^{q}}{\theta^{q}}\right) u_{0} d \theta\right] d t \\
& +\int_{0}^{\infty} e^{-\lambda t}\left[\int_{0}^{t} \int_{0}^{\infty} q \frac{(t-s)^{q-1}}{\theta^{q}} \psi_{q}(\theta) T\left(\frac{(t-s)^{q}}{\theta^{q}}\right) h(s) d \theta d s\right] d t .
\end{aligned}
$$

By the definition of Laplace transforms and the convolution theorem, applying Lemma 2.6 and the inverse Laplace transforms on the above equations, then one can derive that

$$
\begin{aligned}
u(t)= & \mathcal{L}^{-1}\left[\lambda^{q-1}\right] * \mathcal{L}^{-1}\left[\int_{0}^{\infty} e^{-\lambda t}\left[\int_{0}^{\infty} q \frac{t^{q-1}}{\theta^{q}} \psi_{q}(\theta) T\left(\frac{t^{q}}{\theta^{q}}\right) u_{0} d \theta\right] d t\right] \\
& +\mathcal{L}^{-1}\left[\int_{0}^{\infty} e^{-\lambda t}\left(\int_{0}^{t} \int_{0}^{\infty} q \frac{(t-s)^{q-1}}{\theta^{q}} \psi_{q}(\theta) T\left(\frac{(t-s)^{q}}{\theta^{q}}\right) h(s) d \theta d s\right) d t\right] \\
= & \frac{t^{-q}}{\Gamma(1-q)} *\left[\int_{0}^{\infty} q \frac{t^{q-1}}{\theta^{q}} \psi_{q}(\theta) T\left(\frac{t^{q}}{\theta^{q}}\right) u_{0} d \theta\right] \\
& +\int_{0}^{t} \int_{0}^{\infty} q \frac{(t-s)^{q-1}}{\theta^{q}} \psi_{q}(\theta) T\left(\frac{(t-s)^{q}}{\theta^{q}}\right) h(s) d \theta d s \\
= & \int_{0}^{t} \int_{0}^{\infty} \frac{q}{\Gamma(1-q)} s^{-q} \frac{(t-s)^{q-1}}{\theta^{q}} \psi_{q}(\theta) T\left(\frac{(t-s)^{q}}{\theta^{q}}\right) u_{0} d \theta d s+(\mathcal{W h})(t) \\
= & \left(\Upsilon u_{0}\right)(t)+(\mathcal{W h})(t) .
\end{aligned}
$$

Since

$$
\left\|\left(\Upsilon u_{0}\right)(t)+(\mathcal{W} h)(t)\right\|_{\omega} \leq\left\|\left(\Upsilon u_{0}\right)(t)\right\|_{\omega}+\|(\mathcal{W} h)(t)\|_{\omega} \leq\left\|u_{0}\right\|_{\omega}+\frac{1}{\omega} \frac{1}{\Gamma(q+1)}\|h\|_{b \omega}
$$

then

$$
\left\|\Upsilon u_{0}+\mathcal{W} h\right\|_{b \omega} \leq\left\|u_{0}\right\|_{\omega}+\frac{1}{\omega} \frac{1}{\Gamma(q+1)}\|h\|_{b \omega}
$$

Hence, $u \in B C(J, E)$. Then we complete the proof.

\section{Main results}

In this section, we will present the existence theorem for the abstract fractional differential equation on the half-line. In order to prove our main result, we need the following facts and lemmas. 
Lemma 3.1 Let $h \in B C(J, E)$ and $u_{0} \in D(A)$. Let $0<\sum_{i=1}^{\infty} \sigma_{i}<1$. Then the linear fractional evolution equation

$$
\left\{\begin{array}{l}
{ }^{C} D_{0+}^{q} u(t)=A u(t)+h(t), \quad t \in(0,+\infty), \\
u(0)=\sum_{i=1}^{\infty} \sigma_{i} u\left(\tau_{i}\right)
\end{array}\right.
$$

has a unique solution $u \in B C(J, E)$ of the following form:

$$
\begin{aligned}
u(t) & \\
= & \left(\Theta_{A} h\right)(t) \\
:= & \left(\Upsilon\left[(I-\mathcal{R})^{-1} \sum_{i=1}^{\infty} \sigma_{i}(\mathcal{W} h)\left(\tau_{i}\right)\right]\right)(t)+(\mathcal{W} h)(t) \\
= & \int_{0}^{1} \int_{0}^{\infty} \frac{q}{\Gamma(1-q)} \frac{\psi_{q}(\theta)}{\theta^{q}} \tau^{-q}(1-\tau)^{q-1} T\left(t^{q} \frac{(1-\tau)^{q}}{\theta^{q}}\right) \\
& \times\left[(I-\mathcal{R})^{-1}\left(\sum_{i=1}^{\infty} \sigma_{i}(\mathcal{W} h)\left(\tau_{i}\right)\right)\right] d \theta d \tau \\
& +\int_{0}^{t} \int_{0}^{\infty} q \frac{\psi_{q}(\theta)}{\theta^{q}}(t-s)^{q-1} T\left(\frac{(t-s)^{q}}{\theta^{q}}\right) h(s) d \theta d s .
\end{aligned}
$$

Also, $\Theta_{A}$ is a linear operator on the Banach space BC(J,E) and

$$
\left\|\Theta_{A}\right\|_{b \omega} \leq \frac{\chi}{\omega}
$$

Proof In view of Lemma 2.7, one can obtain

$$
u\left(\tau_{i}\right)=\int_{0}^{\tau_{i}} \int_{0}^{\infty}\left[q \frac{\left(\tau_{i}-s\right)^{q-1}}{\theta^{q}} \psi_{q}(\theta) T\left(\frac{\left(\tau_{i}-s\right)^{q}}{\theta^{q}}\right)\left(\frac{s^{-q}}{\Gamma(1-q)} u(0)+h(s)\right)\right] d \theta d s
$$

From $u(0)=\sum_{i=1}^{\infty} \sigma_{i} u\left(\tau_{i}\right)$, we have

$$
\begin{aligned}
u(0)= & \sum_{i=1}^{\infty} \sigma_{i} \int_{0}^{\tau_{i}} \int_{0}^{\infty} \frac{q}{\Gamma(1-q)} \frac{\psi_{q}(\theta)}{\theta^{q}} s^{-q}\left(\tau_{i}-s\right)^{q-1} T\left(\frac{\left(\tau_{i}-s\right)^{q}}{\theta^{q}}\right) u(0) d \theta d s \\
& +\sum_{i=1}^{\infty} \sigma_{i}(\mathcal{W} h)\left(\tau_{i}\right) \\
= & \sum_{i=1}^{\infty} \sigma_{i} \int_{0}^{1} \int_{0}^{\infty} \frac{q}{\Gamma(1-q)} \frac{\psi_{q}(\theta)}{\theta^{q}} \tau^{-q}(1-\tau)^{q-1} T\left(\tau_{i}^{q} \frac{(1-\tau)^{q}}{\theta^{q}}\right) u(0) d \theta d \tau \\
& +\sum_{i=1}^{\infty} \sigma_{i}(\mathcal{W} h)\left(\tau_{i}\right) \\
= & \mathcal{R} u(0)+\sum_{i=1}^{\infty} \sigma_{i}(\mathcal{W} h)\left(\tau_{i}\right) .
\end{aligned}
$$


Therefore,

$$
(I-\mathcal{R}) u(0)=\sum_{i=1}^{\infty} \sigma_{i}(\mathcal{W} h)\left(\tau_{i}\right)
$$

So, we obtain

$$
u(0)=(I-\mathcal{R})^{-1}\left(\sum_{i=1}^{\infty} \sigma_{i}(\mathcal{W} h)\left(\tau_{i}\right)\right)
$$

Then (15) follows.

By (15), one has

$$
\begin{aligned}
\| & \left(\Theta_{A} h\right)(t) \|_{\omega} \\
\leq & \int_{0}^{1} \int_{0}^{\infty} \frac{q}{\Gamma(1-q)} \frac{\psi_{q}(\theta)}{\theta^{q}} \tau^{-q}(1-\tau)^{q-1}\left\|T\left(t^{q} \frac{(1-\tau)^{q}}{\theta^{q}}\right)\right\|_{\omega} \\
& \times\left\|(I-\mathcal{R})^{-1}\left(\sum_{i=1}^{\infty} \sigma_{i}(\mathcal{W} h)\left(\tau_{i}\right)\right)\right\|_{\omega} d \theta d \tau+\|(\mathcal{W} h)(t)\|_{\omega} \\
\leq & \int_{0}^{1} \int_{0}^{\infty} \frac{q}{\Gamma(1-q)} \frac{\psi_{q}(\theta)}{\theta^{q}} \tau^{-q}(1-\tau)^{q-1} e^{-\omega \frac{t^{q}(1-\tau) q^{q}}{\theta^{q}}}\left\|(I-\mathcal{R})^{-1}\right\|_{\omega} \\
& \times\left\|\sum_{i=1}^{\infty} \sigma_{i}(\mathcal{W} h)\left(\tau_{i}\right)\right\| d \theta d \tau+\|(\mathcal{W} h)(t)\|_{\omega} \\
\leq & \frac{1}{1-\sum_{i=1}^{\infty} \sigma_{i}} \frac{\sum_{i=1}^{\infty} \sigma_{i}}{\omega} \frac{1}{\Gamma(q+1)}\|h\|_{b \omega}+\frac{1}{\omega} \frac{1}{\Gamma(q+1)}\|h\|_{b \omega} \\
= & \frac{1}{\omega} \frac{1}{\Gamma(q+1)} \frac{1}{1-\sum_{i=1}^{\infty} \sigma_{i}}\|h\|_{b \omega} \\
= & \frac{\chi}{\omega}\|h\|_{b \omega} .
\end{aligned}
$$

Therefore,

$$
\left\|\Theta_{A} h\right\|_{b \omega} \leq \frac{\chi}{\omega}\|h\|_{b \omega}
$$

In consequence, we have the following lemma.

Lemma 3.2 Let $0<\sum_{i=1}^{\infty} \sigma_{i}<1$. Then problem (1) can be written as the following equivalent integral equation:

$$
u(t)=\operatorname{Tu}(t):=\left(\Theta_{A} f\right)(t)=\left(\Upsilon\left[(I-\mathcal{R})^{-1} \sum_{i=1}^{\infty} \sigma_{i}(\mathcal{W} f)\left(\tau_{i}\right)\right]\right)(t)+(\mathcal{W} f)(t)
$$

Now, we give the proofs of the main result on the existence of positive solutions to problem (1) and (2) in the following. 
Proof of Theorem 1.1 Take two arbitrary elements $u$ and $v$ in Banach $B C(J, E)$. From Lemmas 2.4, 2.6 and 3.2, by the condition $(F)$, we get

$$
\begin{aligned}
\|T u(t)-T v(t)\|_{\omega^{*}} & \|\left(\Upsilon\left[(I-\mathcal{R})^{-1} \sum_{i=1}^{\infty} \sigma_{i} \mathcal{W}\left[f\left(\tau_{i}, u\left(r\left(\tau_{i}\right)\right)\right)-f\left(\tau_{i}, v\left(r\left(\tau_{i}\right)\right)\right)\right]\right]\right)(t) \\
= & +\mathcal{W}[f(t, u(r(t)))-f(t, v(r(t)))] \|_{\omega^{*}} \\
\leq & \left\|\left(\Upsilon\left[(I-\mathcal{R})^{-1} \sum_{i=1}^{\infty} \sigma_{i} \mathcal{W}\left[f\left(\tau_{i}, u\left(r\left(\tau_{i}\right)\right)\right)-f\left(\tau_{i}, v\left(r\left(\tau_{i}\right)\right)\right)\right]\right]\right)(t)\right\|_{\omega^{*}} \\
& +\|\mathcal{W}[f(t, u(r(t)))-f(t, v(r(t)))]\|_{\omega^{*}} \\
\leq & \frac{1}{\omega} \frac{1}{\Gamma(q+1)}\left[\frac{1}{1-\sum_{i=1}^{\infty} \sigma_{i}} \sum_{i=1}^{\infty} \sigma_{i}\left\|f\left(\tau_{i}, u\left(r\left(\tau_{i}\right)\right)\right)-f\left(\tau_{i}, v\left(r\left(\tau_{i}\right)\right)\right)\right\|_{\omega^{*}}\right. \\
& \left.+\|f(t, u(r(t)))-f(t, v(r(t)))\|_{\omega^{*}}\right] \\
\leq & \frac{\mathcal{M}}{\omega} \frac{1}{\Gamma(q+1)}\left[\frac{\sum_{i=1}^{\infty} \sigma_{i}\left\|u\left(r\left(\tau_{i}\right)\right)-v\left(r\left(\tau_{i}\right)\right)\right\|_{\omega^{*}}}{1-\sum_{i=1}^{\infty} \sigma_{i}}\|u(r(t))-v(r(t))\|_{\omega^{*}}\right] \\
\leq & \frac{\mathcal{M}}{\omega} \frac{1}{\Gamma(q+1)}\left[\frac{\sum_{i=1}^{\infty} \sigma_{i}}{1-\sum_{i=1}^{\infty} \sigma_{i}}+1\right]\|u-v\|_{b \omega^{*}} \\
= & \frac{\mathcal{M}}{\omega} \frac{1}{\Gamma(q+1)} \frac{1}{1-\sum_{i=1}^{\infty} \sigma_{i}}\|u-v\|_{b \omega^{*} .}
\end{aligned}
$$

Thus,

$$
\|T u-T v\|_{b \omega^{*}} \leq \mathcal{M} \frac{\chi}{\omega}\|u-v\|_{b \omega^{*}}
$$

which implies that problem (1) has a unique mild solution.

Proof of Theorem 1.2 In the following, similar to the methods used in [27], we will deduce the result of Theorem 1.2 by the monotone iterative method. The proof is divided into four steps.

Step 1: First of all, consider an abstract fractional differential equation as follows:

$$
\left\{\begin{array}{l}
{ }^{C} D_{0+}^{q} u(t)=A u(t)+d_{1} u(t)+f_{0}(t), \quad t \in(0,+\infty), \\
u(0)=\sum_{i=1}^{\infty} \sigma_{i} u\left(\tau_{i}\right) .
\end{array}\right.
$$

Obviously, $\left\{e^{d_{1} t} T(t)\right\}_{t \geq 0}$ is a uniformly exponentially stable $C_{0}$-semigroup on Banach $E$ generated by $A+d_{1} I$, which is positive with the growth bound $d_{1}+\omega_{0}\left(d_{1}+\omega_{0}<0\right)$. Applying Lemma 3.1, equation (17) has a unique mild solution $\phi_{0} \in B C(J, E)$ and $\phi_{0} \geq \theta$ due to $f_{0}(t) \geq \theta, t \in J$. 
Step 2: Letting $g \in B C(J, E)$, consider the following abstract fractional differential equation:

$$
\left\{\begin{array}{l}
{ }^{C} D_{0+}^{q} u(t)+d_{2} u(t)=A u(t)+g(t), \quad t \in(0,+\infty) . \\
u(0)=\sum_{i=1}^{\infty} \sigma_{i} u\left(\tau_{i}\right) .
\end{array}\right.
$$

It is easy to see that $A-d_{2} I$ generates a uniformly exponentially stable $C_{0}$-semigroup $\left\{e^{-d_{2} t} T(t)\right\}_{t \geq 0}$ on Banach $E$. Also, it is positive with the growth bound $-d_{2}+\omega_{0}$ $\left(-d_{2}+\omega_{0}<0\right)$.

According to Lemma 3.1, we can get that problem (18) has a unique mild solution $u=$ $\Theta_{A-d_{2} I} g$, where $\Theta_{A-d_{2} I}: B C(J, E) \longrightarrow B C(J, E)$ is a positive bounded linear operator (similar to the operator $\Theta_{A}$ ) with the property that

$$
\left\|\Theta_{A-d_{2} I}\right\|_{b \omega} \leq \frac{\chi}{d_{2}-\omega_{0}}, \quad \omega=d_{2}-\omega_{0} .
$$

Then $\phi_{0}$ is the mild solution of problem (18) for $g=f_{0}+d_{1} \phi_{0}+d_{2} \phi_{0}$, thus

$$
\phi_{0}=\Theta_{A-d_{2} I}\left(f_{0}+d_{1} \phi_{0}+d_{2} \phi_{0}\right) .
$$

Step 3: We will prove the existence of a mild positive solution of problem (2).

Take $F(u)=f(t, u)+d_{2} u$. Evidently, $F(\theta)=f(t, \theta)=f_{0}(t) \geq \theta$ and $F: B C(J, E) \longrightarrow B C(J, E)$ is continuous due to condition $(H 1),(H 1)$ and the normality of the cone $P_{B}$.

By condition $(H 1)$, then

$$
F(y)-F(x)=f(t, y)+d_{2} y-f(t, x)-d_{2} x=f(t, y)-f(t, x)+d_{2}(y-x) \geq \theta, \quad \theta \leq x \leq y,
$$

which implies that $F$ is an increasing operator on the positive cone $P$.

Let $\varphi_{0}=\theta$ and $\mathcal{O}=\Theta_{A-d_{2} I} \circ F$. It is easy to notice that the fixed point of $\mathcal{O}$ is the mild solution of problem (2). Now, our next step is to prove that the operator $\mathcal{O}$ has at least one fixed point.

Define two sequences

$$
\phi_{n}=\mathcal{O}\left(\phi_{n-1}\right), \quad n=1,2,3, \ldots,
$$

and

$$
\varphi_{n}=\mathcal{O}\left(\varphi_{n-1}\right), \quad n=1,2,3, \ldots
$$

From condition $(H 1)$, one can obtain

$$
f\left(t, \phi_{0}(t)\right)-f(t, \theta) \leq d_{1} \phi_{0}(t)
$$

then

$$
f\left(t, \phi_{0}(t)\right) \leq d_{1} \phi_{0}(t)+f_{0}(t)
$$


Thus,

$$
F\left(\phi_{0}\right)=f\left(t, \phi_{0}(t)\right)+d_{2} \phi_{0}(t) \leq d_{1} \phi_{0}(t)+d_{2} \phi_{0}(t)+f_{0}(t) .
$$

Therefore, we can get

$$
\theta \leq f_{0}(t)=F(\theta) \leq F\left(\phi_{0}\right) \leq d_{1} \phi_{0}+d_{2} \phi_{0}+f_{0} .
$$

For the positivity of the linear bounded operator $\Theta_{A-d_{2} I}$, by (19) and (22), one can get

$$
\theta \leq \Theta_{A-d_{2} I} \circ F(\theta)=\mathcal{O}(\theta) \leq \Theta_{A-d_{2} I} \circ F\left(\phi_{0}\right)=\mathcal{O}\left(\phi_{0}\right) \leq \Theta_{A-d_{2} I}\left(d_{1} \phi_{0}+d_{2} \phi_{0}+f_{0}\right)=\phi_{0},
$$

which shows that

$$
\theta=\varphi_{0} \leq \phi_{1} \leq \phi_{0} .
$$

Since $\mathcal{O}$ is an increasing operator on the order interval $\left[\theta, \phi_{0}\right]$, in view of the definition of $\mathcal{O}$ and (23), we can get two sequences $\left\{\phi_{n}\right\}$ and $\left\{\varphi_{n}\right\}(n=0,1,2,3, \ldots)$ such that

$$
\theta=\varphi_{0} \leq \varphi_{1} \leq \varphi_{2} \leq \cdots \leq \varphi_{n} \leq \cdots \leq \phi_{n} \leq \cdots \leq \phi_{2} \leq \phi_{1} \leq \phi_{0} .
$$

From condition $(H 1)$, we have

$$
\begin{aligned}
\theta \leq \phi_{n}-\varphi_{n} & =\mathcal{O}\left(\phi_{n-1}\right)-\mathcal{O}\left(\varphi_{n-1}\right) \\
& =\Theta_{A-d_{2} I} \circ F\left(\phi_{n-1}\right)-\Theta_{A-d_{2} I} \circ F\left(\varphi_{n-1}\right) \\
& =\Theta_{A-d_{2} I}\left[f\left(\cdot, \phi_{n-1}\right)+d_{2} \phi_{n-1}-f\left(\cdot, \varphi_{n-1}\right)-d_{2} \varphi_{n-1}\right] \\
& \leq\left(d_{1}+d_{2}\right) \Theta_{A-d_{2} I}\left(\phi_{n-1}-\varphi_{n-1}\right) .
\end{aligned}
$$

Then

$$
\theta \leq \phi_{n}-\varphi_{n} \leq\left(d_{1}+d_{2}\right)^{n} \Theta_{A-d_{2} I}\left(\phi_{0}-\varphi_{0}\right)=\left(d_{1}+d_{2}\right)^{n} \Theta_{A-d_{2} I}^{n}\left(\phi_{0}\right) .
$$

Since the cone $P_{B}$ is normal with the normal constant $N$, by virtue of condition $(H 2)$, one can get

$$
\begin{aligned}
\left\|\phi_{n}-\varphi_{n}\right\|_{b \omega} & \leq N\left(d_{1}+d_{2}\right)^{n}\left\|\Theta_{A-d_{2} I}^{n}\left(\phi_{0}\right)\right\|_{b \omega} \\
& \leq N\left(d_{1}+d_{2}\right)^{n}\left\|\Theta_{A-d_{2} I}^{n}\right\|_{b \omega}\left\|\phi_{0}\right\|_{b \omega} \\
& \leq N\left(d_{1}+d_{2}\right)^{n}\left\|\Theta_{A-d_{2} I}\right\|_{b \omega}^{n}\left\|\phi_{0}\right\|_{b \omega} \\
& \leq N\left(d_{1}+d_{2}\right)^{n}\left(\frac{\chi}{d_{2}-\omega_{0}}\right)^{n}\left\|\phi_{0}\right\|_{b \omega} \\
& =N\left(\frac{\chi\left(d_{1}+d_{2}\right)}{d_{2}-\omega_{0}}\right)^{n}\left\|\phi_{0}\right\|_{b \omega} \rightarrow 0, \quad n \rightarrow+\infty .
\end{aligned}
$$

Therefore, by a method similar to the nested interval one, by (24), there exists unique $u^{*} \in \bigcap_{n=1}^{\infty}\left[\varphi_{n}, \phi_{n}\right]$ such that $u^{*}=\lim _{n \rightarrow \infty} \phi_{n}=\lim _{n \rightarrow \infty} \varphi_{n}$. 
Taking limit of $n \rightarrow \infty$ both on (20) and (21), we can obtain that

$$
u^{*}=\mathcal{O}\left(u^{*}\right)
$$

which shows that $u^{*}$ is the fixed point of $\mathcal{O}$. Hence, $u^{*}$ is a mild positive solution of problem (2).

Step 4: In the last step, we will prove the uniqueness of the mild solution for problem (2).

By reduction to absurdity, assume that $u_{1}^{*}$ and $u_{2}^{*}$ are two different positive mild solutions for the fractional evolution equation (2), so $\left\|u_{1}^{*}-u_{2}^{*}\right\|_{b \omega}>0$.

Replace $\phi_{0}$ by $u_{1}^{*}$ and $u_{2}^{*}$ in (20), respectively. Following the same steps as above, for each $u_{i}^{*}(i=1,2)$, we can get that $u_{i}^{*}=\mathcal{O}\left(u_{i}^{*}\right),\left\|u_{i}^{*}-\varphi_{n}\right\|_{b \omega} \rightarrow 0(n \rightarrow \infty)$ and $\phi_{n}=u_{i}^{*}$ for each $n \in \mathbb{N}(i=1,2)$. Therefore,

$$
0<\left\|u_{1}^{*}-u_{2}^{*}\right\|_{b \omega} \leq\left\|u_{1}^{*}-\varphi_{n}\right\|_{b \omega}+\left\|u_{2}^{*}-\varphi_{n}\right\|_{b \omega} \rightarrow 0, \quad n \rightarrow \infty
$$

which is a contradiction.

Hence, problem (2) has a unique positive solution. The proof is completed.

\section{Examples}

To illustrate our main result, we will present an example. Consider the following partial fractional differential equation.

\section{Example 1}

$$
\begin{cases}\partial_{t}^{q} z(t, x)=\partial_{x}^{2} z(t, x)+F(t, z(t, x)), & t \in[0,+\infty), \\ z(t, 0)=z(t, \pi)=0, & t \in[0,+\infty), \\ z(0, x)=\sum_{i=1}^{\infty} \sigma_{i} z\left(\tau_{i}, x\right), & x \in[0, \pi],\end{cases}
$$

where $\partial_{t}^{q}$ is the Caputo fractional partial derivative of order $q \in(0,1)$.

Set $E=L^{2}([0, \pi], \mathbb{R})$ and $A z=\partial_{x}^{2} z$, according to [33], then $A: D(A) \longrightarrow E$ is a linear operator with domain $D(A)=\left\{u \in E \mid u^{\prime} \in E, u(0)=u(\pi)=0\right\}$. Besides, the operator $A$ generates a uniformly exponentially stable $C_{0}$-semigroup $\{T(t)\}_{t \geq 0}$ with the growth bound $\omega_{0} \leq-1$.

Let $u(t)=z(t, \cdot), f(t, u(t))=F(t, z(t, \cdot))$, then problem (1) can be written as

$$
\left\{\begin{array}{l}
{ }^{C} D_{0+}^{q} u(t)=A u(t)+f(t, u(t)), \quad t \in(0,+\infty), \\
u(0)=\sum_{i=1}^{\infty} \sigma_{i} u\left(\tau_{i}\right) .
\end{array}\right.
$$

Take $q=1 / 2, \sum_{i=1}^{\infty} \sigma_{i}=5 / 6, \tau_{i}>0, i=1,2, \ldots$, then we can get

$$
\chi=\frac{1}{\Gamma(q+1)} \frac{1}{1-\sum_{i=1}^{\infty} \sigma_{i}}=\frac{12}{\sqrt{\pi}} .
$$

Consider the following function:

$$
f(t, x)=\left(-d_{2}+\frac{-\omega_{0}}{\left(1+a^{2}(t)\right)(\chi-1)}\right) x
$$


where $a \in C[0,+\infty)$ is bounded and

$$
d_{1}=2 \omega_{0}, \quad d_{2}=-\left(2+\frac{2}{\chi-1}\right) \omega_{0} .
$$

It is easy for us to certify that

$$
0<\frac{d_{1}+d_{2}}{d_{2}-\omega_{0}}=\frac{2}{3 \chi-1}<\frac{1}{\chi}
$$

Since

$$
-d_{2}-\frac{\omega_{0}}{\chi-1}=\left(2+\frac{2}{\chi-1}\right) \omega_{0}-\frac{\omega_{0}}{\chi-1} \leq 2 \omega_{0}=d_{1}
$$

then, for $\theta \leq x \leq y$,

$$
\begin{aligned}
-d_{2}(y-x) \leq f(t, y)-f(t, x) & =\left[-d_{2}+\frac{-\omega_{0}}{\left(1+a^{2}(t)\right)(\chi-1)}\right](y-x) \\
& \leq\left(-d_{2}-\frac{\omega_{0}}{\chi-1}\right)(y-x) \leq d_{1}(y-x) .
\end{aligned}
$$

Noting that $f(t, \theta)=\theta$. Thereby, $f$ satisfies the conditions of Theorem 1.2. We can conclude that problem (1) has a unique positive mild solution.

\section{Acknowledgements}

This research is supported by the NNSF of China (NO. 71672195), the NNSF of China (NO. 11601508) and the NNSF of China (NO. 71272066).

\section{Competing interests}

The authors declare that they have no competing interests.

\section{Authors' contributions}

All of the authors contributed equally in writing this paper. All authors read and approved the final manuscript.

\section{Author details}

${ }^{1}$ School of Business, Central South University, Changsha, 410083, China. ${ }^{2}$ School of Mathematics, China University of Mining and Technology, Xuzhou, 221116, China.

\section{Publisher's Note}

Springer Nature remains neutral with regard to jurisdictional claims in published maps and institutional affiliations.

Received: 16 July 2017 Accepted: 16 November 2017 Published online: 13 December 2017

\section{References}

1. Kilbas, AA, Srivastava, HM, Trujillo, JJ: Theory and Applications of Fractional Differential Equations. Elsevier, Amsterdam (2006)

2. Miller, KS, Ross, B: An Introduction to the Fractional Calculus and Fractional Differential Equations. Wiley, New York (1993)

3. Lakshmikantham, V, Leela, S, Vasundhara Devi, J: Theory of Fractional Dynamic Systems. Cambridge Academic Publishers, Cambridge (2009)

4. Zhou, Y, Ahmadb, B, Alsaedi, A: Existence of nonoscillatory solutions for fractional neutral differential equations. Appl. Math. Lett. 72, 70-74 (2017)

5. Zou, Y, He, G: On the uniqueness of solutions for a class of fractional differential equations. Appl. Math. Lett. 74, 68-73 (2017)

6. Cabada, A, Kisela, T: Existence of positive periodic solutions of some nonlinear fractional differential equations. Commun. Nonlinear Sci. Numer. Simul. 50, 51-67 (2017)

7. Raheem, A, Maqbul, MD: Oscillation criteria for impulsive partial fractional differential equations. Comput. Math. Appl. 73, 1781-1788 (2017) 
8. Henderson, J, Luca, R: Systems of Riemann-Liouville fractional equations with multi-point boundary conditions. Appl. Math. Comput. 309, 303-323 (2017)

9. Ahmad, B, Alsaedi, A, Garout, D: Existence results for Liouville-Caputo type fractional differential equations with nonlocal multi-point and sub-strips boundary conditions. Comput. Math. Appl. (2017, in press). 10.1016/j.camwa.2016.04.015

10. Dhifli, A, Khamessi, B: Existence and boundary behavior of positive solution for a Sturm-Liouville fractional problem with $p$-Laplacian. J. Fixed Point Theory Appl. 2, 1-22 (2017)

11. Ahmada, B, Ntouyas, SK, Alsaedi, A: On a coupled system of fractional differential equations with coupled nonlocal and integral boundary conditions. Chaos Solitons Fractals 83, 234-241 (2016)

12. Bayour, B, Torres, DFM: Existence of solution to a local fractional nonlinear differential equation. J. Comput. Appl. Math. 312, 127-133 (2017)

13. Mei, ZD, Peng, JG, Gao, JH: General fractional differential equations of order $\alpha \in(1,2)$ and type $\xi_{i} \in[0,1]$ in Banach spaces. Semigroup Forum 94, 712-737 (2017)

14. El-Borai, MM: Some probability densities and fundamental solutions of fractional evolution equations. Chaos Solitons Fractals 14, 433-440 (2002)

15. Zhou, Y, Jiao, F: Nonlocal Cauchy problem for fractional evolution equations. Nonlinear Anal., Real World Appl. 11, 4465-4475 (2010)

16. Wang, J, Zhou, Y: A class of fractional evolution equations and optimal controls. Nonlinear Anal., Real World Appl. 12, 262-272 (2011)

17. Chen, P, Zhang, X, Li, Y: Study on fractional non-autonomous evolution equations with delay. Comput. Math. Appl. 73, 794-803 (2017)

18. Chen, $\mathrm{P}, \mathrm{Li}, \mathrm{Y}, \mathrm{Li}, \mathrm{Q}$ : Existence of mild solutions for fractional evolution equations with nonlocal initial conditions. Ann. Pol. Math. 110, 13-24 (2014)

19. Chen, $P, L i, Y, Z$ hang, $X$ : On the initial value problem of fractional stochastic evolution equations in Hilbert spaces. Commun. Pure Appl. Anal. 14, 1817-1840 (2015)

20. Wang, R, Ma, Q: Some new results for multi-valued fractional evolution equations. Appl. Math. Comput. 257, 285-294 (2015)

21. Zhao, J, Wang, R: Mixed monotone iterative technique for fractional impulsive evolution equations. Miskolc Math Notes 17, 683-696 (2016)

22. Jabeena, T, Lupulescu, V: Existence of mild solutions for a class of non-autonomous evolution equations with nonlocal initial conditions. J. Nonlinear Sci. Appl. 10, 141-153 (2017)

23. Zhou, Y, Shen, $X H$, Zhang, L: Cauchy problem for fractional evolution equations with Caputo derivative. Eur. Phys. J. Spec. Top. 222, 1749-1765 (2013)

24. Byszewski, L: Theorems about existence and uniqueness of solutions of a semi-linear evolution nonlocal Cauchy problem. J. Math. Anal. Appl. 162, 494-505 (1991)

25. Byszewski, L, Lakshmikantham, V: Theorems about existence and uniqueness of a solution of a nonlocal abstract Cauchy problem in a Banach space. Appl. Anal. 40, 11-19 (1991)

26. Byszewski, L: Existence and uniqueness of a classical solution to a functional-differential abstract nonlocal Cauchy problem. J. Appl. Math. Stoch. Anal. 12, 91-97 (1999)

27. Chen, $P, L i, Y, Z$ Zhang, $X$ : Existence and uniqueness of positive mild solutions for nonlocal evolution equations. Positivity 19, 927-939 (2015)

28. Deimling, K: Nonlinear Functional Analysis. Springer, New York (1985)

29. Guo, D, Lakshmikantham, V: Nonlinear Problems in Abstract Cone. Academic Press, Orlando (1988)

30. Pazy, A: Semigroups of Linear Operators and Applications to Partial Differential Equations. Springer, New York (1983)

31. Engel, K, Nagel, R: One-Parameter Semigroups for Linear Evolution Equations. Springer, New York (1995)

32. Mainardi, F, Paradisi, P, Gorenflo, R: Probability distributions generated by fractional diffusion equations. In: Kertesz, J, Kondor, I (eds.) Econophysics: An Emerging Science. Kluwer, Dordrecht (2000)

33. Hernandez, E, Sakthivel, R, Tanaka, AS: Existence results for impulsive evolution differential equations with state-dependent delay. Electron. J. Differ. Equ. 2008, 28 (2008)

\section{Submit your manuscript to a SpringerOpen ${ }^{\circ}$ journal and benefit from:}

- Convenient online submission

- Rigorous peer review

- Open access: articles freely available online

- High visibility within the field

- Retaining the copyright to your article

Submit your next manuscript at $\gg$ springeropen.com 